\title{
A BEAM PROFILE MONITOR FOR RARE ISOTOPES IN ACCELERATOR MASS SPECTROMETRY: PRELIMINARY MEASUREMENTS
}

\author{
F Taccetti • L Carraresi • M E Fedi ${ }^{1} \bullet$ M Manetti • P Mariani • G Tobia • P A Mandò \\ INFN Sezione di Firenze e Dipartimento di Fisica e Astronomia dell’Università di Firenze, via Sansone 1, 50019 Sesto \\ Fiorentino (Fi), Italy.
}

\begin{abstract}
In accelerator systems, beam lines are generally equipped with diagnostic elements, such as Faraday cups and beam profile monitors (BPM), to optimize beam transport. These diagnostic elements, or at least commercial ones, are designed to only work with continuous beams, and their typical maximum sensitivity is about few tens of pA. Thus, in the case of diagnosis of rare isotope beams in accelerator mass spectrometry (AMS), Faraday cups and BPMs are not suitable on the high-energy side of the tandem accelerator, after energy-mass-charge analysis. For example, in ${ }^{14} \mathrm{C} A M S$, even for a modern sample, the expected counting rate is a few tens of $\mathrm{Hz}$; in these conditions, a commercial BPM cannot be used. On the other hand, checking the shape and the position of the rare isotope beam hitting the detector can be important in order to better identify signals in the detector itself, thus also helping in reducing the measurement background.
\end{abstract}

This paper presents a prototype BPM especially designed for low-intensity beams. The BPM is based on a multiwire proportional chamber characterized by 2 grids of anode wires, oriented perpendicular to each other in order to measure both the $x$ and the $y$ coordinates of the particle impact point. Details about the design and the electronics of the device are given, and the first test measurements are discussed.

\section{INTRODUCTION}

Accelerator mass spectrometry (AMS) is a well-established technique used to measure the abundance of rare isotopes, including ${ }^{14} \mathrm{C},{ }^{10} \mathrm{Be},{ }^{129} \mathrm{I}$, and many others. In AMS, a loss in beam transmission due to bad transport can lead to systematic errors in the isotopic ratio measurement. Thus, the measurement of beam characteristics, such as the current, shape, and position on the transversal plane, is of crucial importance in order to optimize beam transport. In tandem-based AMS systems, especially on the low-energy side, AMS beam lines are usually equipped with diagnostic stations, typically consisting of a Faraday cup, to measure particle currents, and a beam profile monitor (BPM), to reconstruct shape and position of the beam. Commercial BPMs (at least those used in low-energy electrostatic accelerators) are based on the measurement of the current due to the electrons created when the incident ions impinge on a moving wire. They are characterized by 1 or more wires that during their motion, intercept the beam in 2 directions, one perpendicular to the other, in order to measure the spatial distribution of the particles in the plane that is transversal to their direction of motion. Their sensitivity is thus typically limited to few tens of pA (http://www.pelletron.com; http://www.highvolteng.com), which is extremely good when measuring beam characteristics on the low-energy side of a tandem accelerator. On the contrary, on the high-energy side, after mass-energy-charge analysis, typical rare isotope beam currents are well below the limit of sensitivity for commercial BPMs (Fedi et al. 2007). However, checking the shape and the position of the rare isotope beam hitting the detector is important during beam tuning, since information on the trajectories of the incoming particles can offer hints to identify the signals in the detector itself. In this way, focusing and aligning of the beam can be improved, thus helping us in minimizing "unwanted" counts. To this end, at LABEC (Laboratorio di Tecniche Nucleari per i Beni Culturali, Florence), a BPM has been developed by modifying a multiwire proportional chamber (MWPC). In the following, the principal features of our prototype and the first test measurements are presented and discussed.

${ }^{1}$ Corresponding author. Email: fedi@fi.infn.it.

(C) 2010 by the Arizona Board of Regents on behalf of the University of Arizona Proceedings of the 20th International Radiocarbon Conference, edited by A J T Jull RADIOCARBON, Vol 52, $\mathrm{Nr} 2-3,2010$, p 272-277 


\section{THE MULTIWIRE PROPORTIONAL CHAMBER USED AS BPM}

The multiwire proportional chamber (Charpak and Sauli 1979) is a particular type of proportional counter that can measure the impact point of the detected particles. The BPM developed at LABEC consists of a central cathode placed between 2 grids of anode wires, oriented perpendicular to each other (see Figure 1), in order to measure both the $x$ and the $y$ coordinates of the particle impact point. Each grid, placed at a distance of $3 \mathrm{~mm}$ from the cathode, is made of 40 gold-plated tungsten wires, having a diameter of $20 \mu \mathrm{m}$, on a pitch of $1 \mathrm{~mm}$. The cathode is a double-sided aluminized Mylar ${ }^{\circledR}$ foil, kept at a negative voltage $(-590 \mathrm{~V})$ with respect to the anode wires. The wires are maintained at ground potential by means of the amplifying system. The entire system is enclosed by 2 Mylar windows, which ensure gas tightness. The detector is filled with heptane $\left(\mathrm{C}_{7} \mathrm{H}_{16}\right)$, continuously flowing and kept at a constant pressure of about 3.5 mbar. As entrance windows, $1.3-\mu \mathrm{m}$-thick Mylar foils were chosen as a good compromise between low beam spread after passing through the device and good mechanical strength of the window itself. Nevertheless, due to passing through Mylar windows and heptane-filled volumes, the beam, which has typically a low value of $\mathrm{MeV} /$ amu, will be strongly degraded in both energy and spatial distribution. Our idea is therefore to mount the device on a mechanism that allows us to insert it on the beam axis only during the beam tuning phases and to retract it during actual AMS measurements.
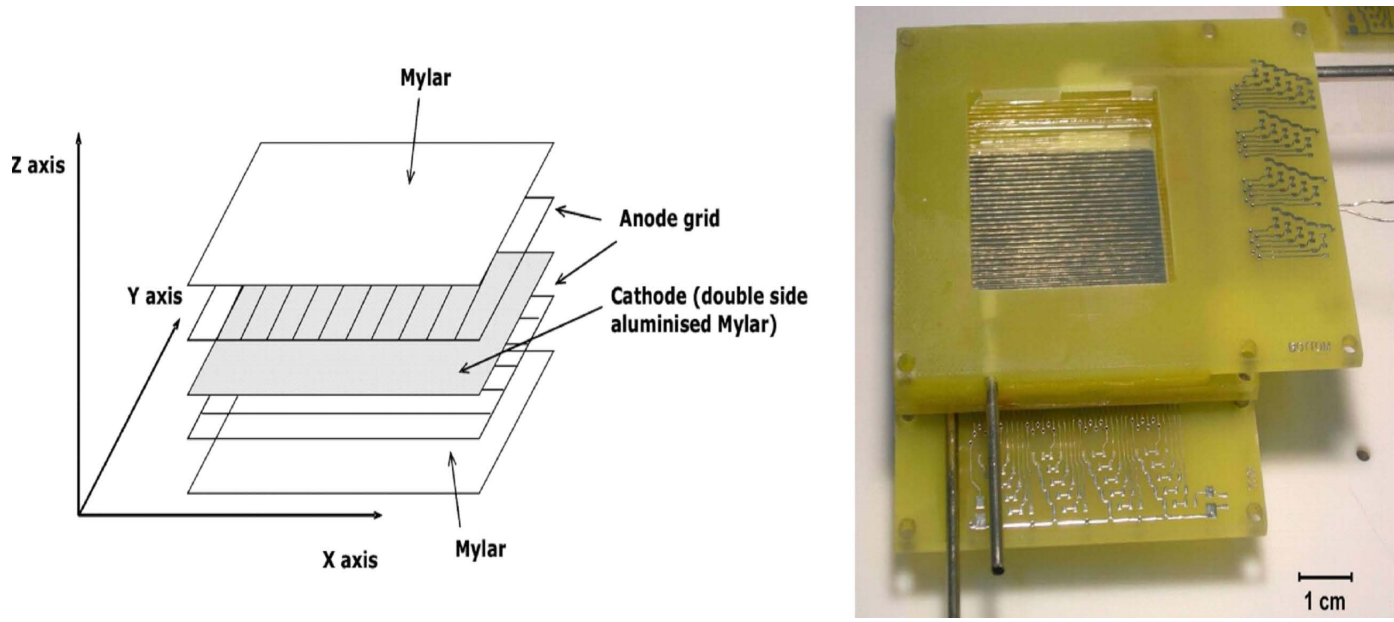

Figure 1 The multiwire proportional chamber used as a beam profile monitor: a) drawing of the designed device (direction of the beam is in the $z$ axis direction); b) photo of the assembled prototype (Mylar ${ }^{\circledR}$ entrance window has not been mounted yet, thus allowing a view at one of the anode grids).

When an ionizing particle hits the MWPC, an avalanche occurs inside it, in both parts of the detector containing the anode grids. A positive signal due to ions is collected to the cathode, while a negative signal develops on the wires interested by the avalanche, both in the grid oriented along the $x$ axis and in the one oriented along the $y$ axis. The impact point of the particle is reconstructed using the anode signals, mixed together to reduce the electronics required. Each wire of the 2 grids is connected to a delay-line chain: signals are collected only at the ends of the chain ( 2 for each of the grids). Each delay-line chain consists of 39 pairs of capacitors (C) and inductors (L), whose values are $47 \mathrm{pF}$ and $150 \mathrm{nH}$, respectively. Each couple provides a delay $\tau=\sqrt{L \cdot C}$ of $\sim 2.7 \mathrm{~ns}$; the end of each of the lines is connected to a preamplifier. The lines have a characteristic impedance of $R_{0}=$ $\sqrt{\frac{L}{C}}=56 \Omega$ 


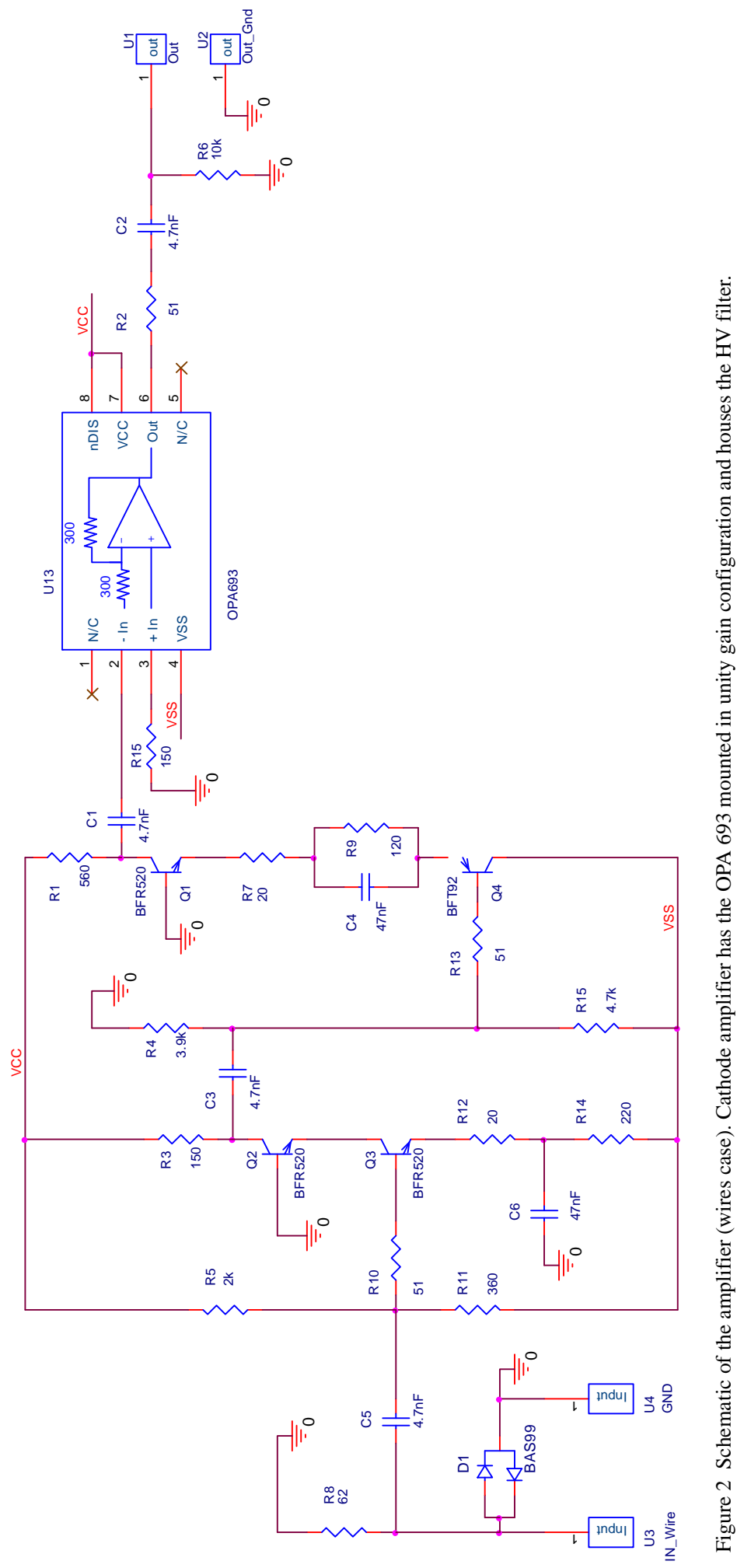


The signal produced in each grid by the ionizing particle reaches the corresponding delay line and travels along it in both directions, until it is collected by the preamplifiers at both ends of the delay line. Depending on the wire hit by the particle, the signal is collected at different times. The impact point can be deduced by measuring any of the following timing differences: $\Delta \mathrm{T}_{\text {left }}$, i.e. difference between collection of signal at cathode and collection of the signal at one end of the delay line; $\Delta \mathrm{T}_{\text {right }}$, i.e. difference between collection of signal at cathode and collection of the signal at the opposite end of the same delay line; or $\Delta \mathrm{T}_{1-\mathrm{r}}$, i.e. difference between collection of signals at the 2 ends of the delay line. This mechanism of timing differences measurement reduces the number of signals coming from the detector to only 5 . The signals coming from the delay lines and from the cathode are amplified by means of custom electronic boards housed on the MWPC support. The amplifiers, shown in Figure 2, are based on double-cascode stages followed by a video amplifier, in unitary gain configuration to drive the line impedance.

The preliminary chain used to acquire timing differences is based on the use of Canberra 454 constant fraction discriminators (CFD) and an Ortec 437A time to amplitude converter (TAC). The use of CFDs, rather than simpler leading edge discriminators, is due to the fact that the amplitude and the rise time of the collected signals can have large variations: actually, both amplitude and rise time depend on which wire is interested by the avalanche, i.e. on the impact point of the primary particle. These variations might introduce systematic effects, just minimized by CFDs.

\section{TEST MEASUREMENTS AND DISCUSSION}

Before installing the new BPM on the high-energy side of our AMS beam line, the prototype was tested using one of the ion beam analysis lines in our laboratory (Mandò 2009). This was done so as not to interfere with "routine" radiocarbon measurements. The beam line, commonly called DEFEL (Taccetti et al. 2003; Mirto and Carraresi 2007), is equipped with a double electrostatic deflector and is capable of chopping the incoming continuous ion beam down to single particle packets, at rates as low as $1 \mathrm{~Hz}$. In this way, we obtain a very low-intensity beam from a continuous beam of the stable isotope ${ }^{12} \mathrm{C}$. At the end of the DEFEL beam line, a large vacuum chamber equipped with a gas system facility (to manage gas-filled devices) and a 2-axial linear stage motion system is present.

In these test measurements, we illuminated the BPM with a ${ }^{12} \mathrm{C}$ beam whose characteristics were almost the same as the ${ }^{14} \mathrm{C}$ beam we usually have during AMS ${ }^{14} \mathrm{C}$ measurements. The beam was extracted from the AMS sputter source and accelerated using a terminal voltage of $2.5 \mathrm{MV}$. The 10$\mathrm{MeV}{ }^{12} \mathrm{C}^{+3}$ ion beam was selected by means of a switching magnet present at the entrance of the DEFEL line and chopped at a frequency of $50 \mathrm{~Hz}$. The final dimensions of the ${ }^{12} \mathrm{C}$ beam were determined by slits placed before the measurement vacuum chamber set to $0.2 \times 0.2 \mathrm{~mm}^{2}$. The main features of the beam used for the tests were checked using a Hamamatsu S3590 silicon detector followed by a Tennelec 171 preamplifier and an Ortec 572 shaper amplifiers. As shown in Figure 3, more than $95 \%$ of the packets hitting the detector are made by a single particle. The BPM was then mounted on the linear stage motion system and its spatial resolution was tested by moving it in steps of $0.25 \mathrm{~mm}$.

Figure 4 shows the response of the BPM at 4 adjoining steps; each peak contains about $10^{4}$ counts and has been fitted with a Gaussian shape to estimate the full width at half maximum (FWHM) of the profile. As indicated in the figure, the FWHM is $\sim 0.2 \mathrm{~mm}$, i.e. of the order of the beam dimensions. Thus, in the present situation, the resolution of the BPM seems to be dependent on the aperture of the slits at the entrance of the vacuum chamber, and we can expect to improve it. 

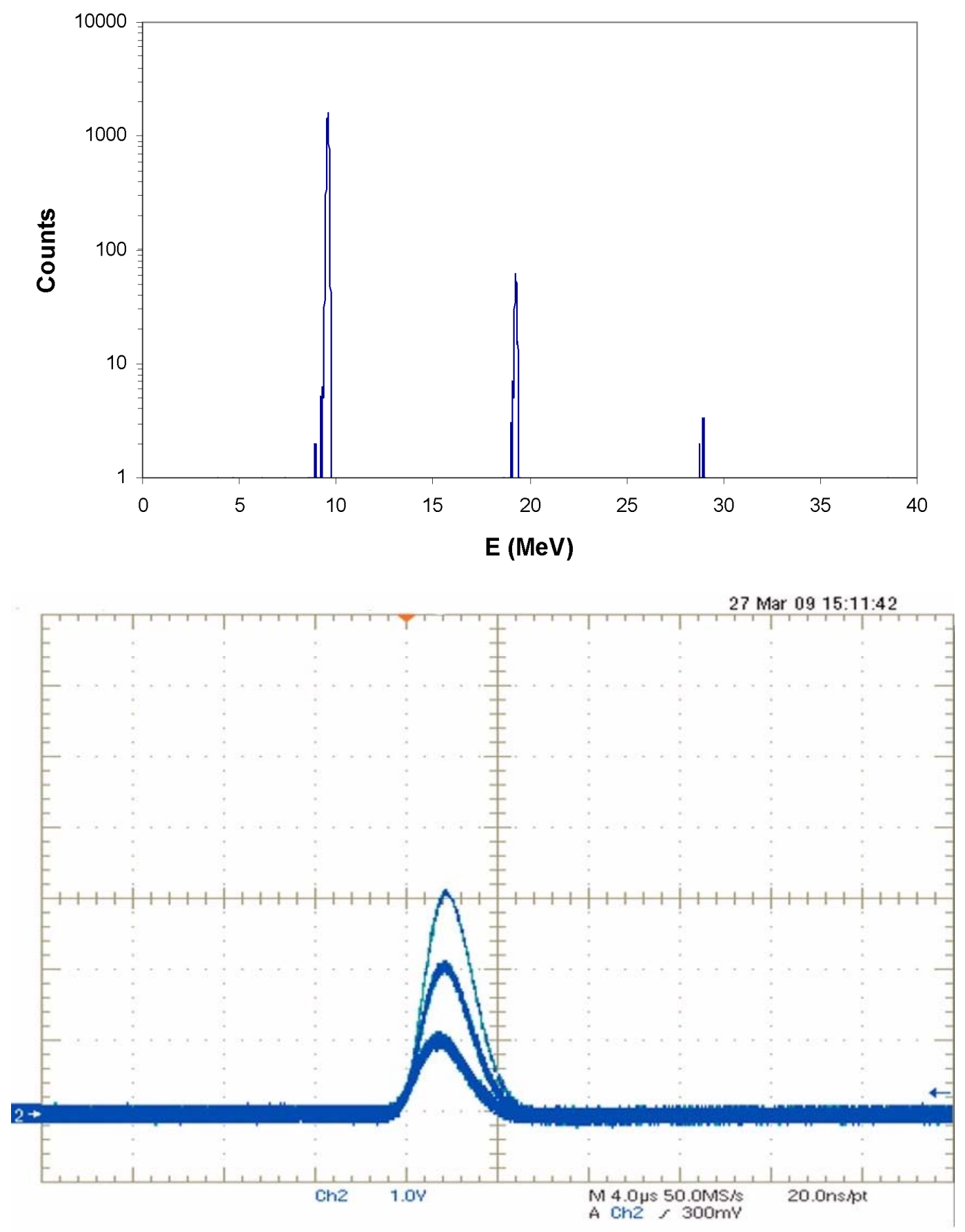

Figure 3 Beam characterization. Above: beam energy peaks are reported. Below: image from the digital oscilloscope acquired in "infinite persistence" mode to enhance packets multiplicity. 


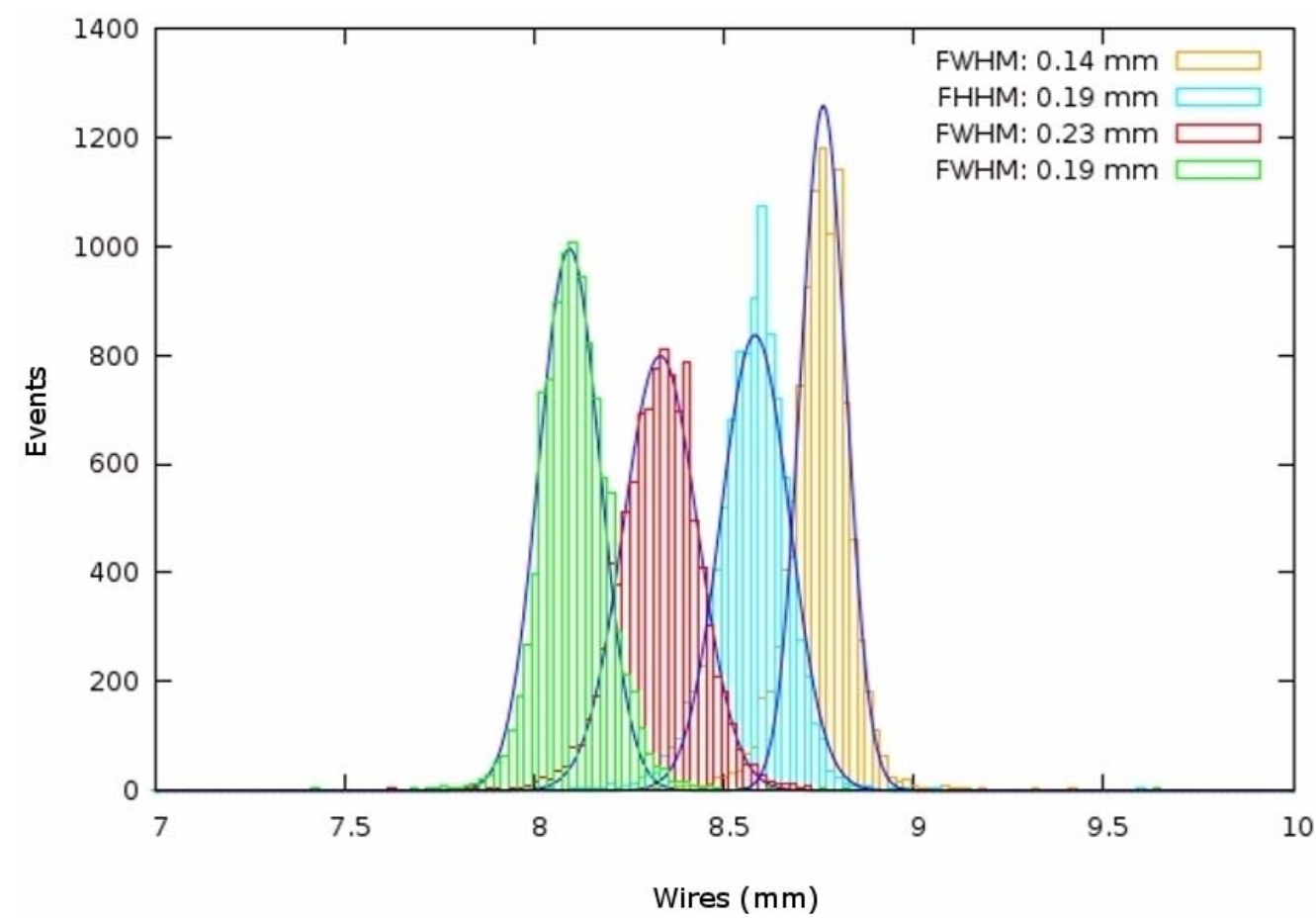

Figure 4 Spectra recorded by moving the BPM in 4 steps of $0.25 \mathrm{~mm}$

On the other hand, one can notice that the peak shapes are quite different at different positions and seem to depend on the impact point of the particle with respect to the wire. Other measurements need to be done to further investigate the charge collection near the wire. Moreover, other tests with a smaller beam spot are planned, as well as efforts to reduce the Mylar windows thickness. Nevertheless, these first measurements are encouraging and even this first version of the beam profile monitor seems to be suitable to meet our goal.

\section{ACKNOWLEDGMENTS}

This work was partially supported by the project ST@RT of Regione Toscana.

\section{REFERENCES}

Charpak G, Sauli F. 1979. Multiwire proportional chambers and drift chambers. Nuclear Instruments and Methods 162(1-3):405-28.

Fedi ME, Cartocci A, Manetti M, Taccetti F, Mandò PA. 2007. The ${ }^{14} \mathrm{C}$ AMS facility at LABEC, Florence. $\mathrm{Nu}$ clear Instruments and Methods in Physics Research B 259(1):18-22.

Mandò PA. 2009. INFN-LABEC, nuclear techniques for cultural heritage and environmental applications. $\mathrm{Nu}$ clear Physics News 19(1):5-12.

Mirto FA, Carraresi L. 2007. The pulsed beam facility at the Tandetron accelerator in Florence. Nuclear Instruments and Methods in Physics Research B 266(10): 2113-6.

Taccetti N, Poggi G, Carraresi L, Bini M, Casini G, Ciaranfi R, Giuntini L, Maurenzig PR, Montecchi M, Olmi A, Pasquali G, Piantelli S, Stefanini AA. 2003. Linear electronics for Si-detectors and its energy calibration for use in heavy ion experiments. Nuclear Instruments and Methods in Physics Research A 496(23):481-95. 